\begin{tabular}{|c|c|c|}
\hline 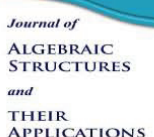 & $\begin{array}{l}\text { Journal of Algebraic Structures and Their Applications } \\
\qquad \text { ISSN: } 2382-9761\end{array}$ & \\
\hline-1 & www.as.yazd.ac.ir & \\
\hline
\end{tabular}

Algebraic Structures and Their Applications Vol. 6 No. 1 ( 2019 ) pp 23-33.

\title{
CARTESIAN CLOSED SUBCATEGORIES OF TOPOLOGICAL FUZZES
}

\author{
MAHBOOBEH AKBARPOUR AND GHASEM MIRHOSSEINKHANI*
}

\begin{abstract}
A category $\mathbf{C}$ is called Cartesian closed provided that it has finite products and for each $\mathbf{C}$-object $A$ the functor $(A \times-): A \rightarrow A$ has a right adjoint. It is well known that the category TopFuzz of all topological fuzzes is both complete and cocomplete, but it is not Cartesian closed. In this paper, we introduce some Cartesian closed subcategories of this category.
\end{abstract}

\section{INTRODUCTION}

A completely distributive complete lattice is called a molecular lattice. In 1992, Wang introduced the concept of topological molecular lattices in terms of closed elements as a generalization of ordinary topological spaces, fuzzy topological spaces and $L$-fuzzy topological spaces in tools of molecules, remote neighborhoods and generalized order homomorphisms [9]. A fuzz is a pair $\left(F,^{\prime}\right)$ consisting of a molecular lattice $F$ and an order reversing involution ' $: F \rightarrow F$, that is, $x \leq y$ if and only if $y^{\prime} \leq x^{\prime}$ and $x^{\prime \prime}=x$ for all $x, y \in F$. A topological fuzz is a triple $\left(F,{ }^{\prime}, \tau\right)$ such that $\left(F,{ }^{\prime}\right)$ is a fuzz and $\tau \subseteq F$ is a topology, i.e., it is closed under finite DOI : $10.29252 /$ as. 2019.1335

MSC(2010): Primary: 06DXX, 06F30

Keywords: Topological fuzz, Exponentiable object, Cartesian closed category.

Received: 30 Jan 2018, Accepted: 10 Sep 2018.

*Corresponding author

(C) 2019 Yazd University. 
meets, arbitrary joins and $0,1 \in \tau$, where 0 and 1 are the smallest and the greatest elements of $F$, respectively. Every element of a topology $\tau$ is called open and every element of $\tau^{\prime}$ is called closed, where $\tau^{\prime}=\left\{a^{\prime} \mid a \in \tau\right\}$.

For two molecular lattices $F$ and $G$, and a mapping $f: F \rightarrow G$ which preserves arbitrary joins, suppose $\widehat{f}$ denote the right adjoint of $f$, then $\widehat{f}: G \rightarrow F$ is defined by $\widehat{f}(y)=\bigvee\{x \in$ $F \mid f(x) \leq y\}$ for every $y \in G$. A map $f:\left(F,{ }^{\prime}\right) \rightarrow\left(G,{ }^{\prime}\right)$ between fuzzes is called an order homomorphism or a fuzz-map in this paper if $f$ preserves arbitrary joins and $\widehat{f}$ preserves ' [i0]. A fuzz-map $f:\left(F,{ }^{\prime}, \tau\right) \rightarrow\left(G,{ }^{\prime}, \mu\right)$ between topological fuzzes is said to be continuous if $b \in \mu$ implies $\widehat{f}(b) \in \tau$.

The category of all fuzzes with fuzz-maps between them is denoted by Fuzz, and the category of all topological fuzzes with continuous fuzz-maps between them is denoted by TopFuzz. It is well known that these categories are both complete and cocomplete, and some categorical structures of them were introduced by many authors [4, 5, 8, 10, 11]. Topological fuzzes are more general frames for studding fuzzy topological spaces. Since the category Top of all topological spaces, as a full subcategory of TopFuzz, is reflective and coreflective [8], this points out the essential difference between the general topological spaces and topological fuzzes on the categorical level.

An object $A$ of a category $\mathbf{C}$ with finite products is called exponentiable if the functor $A \times-: \mathbf{C} \rightarrow \mathbf{C}$ has a right adjoint and it is called cartesian closed provided that every object of $\mathbf{C}$ is exponentiable. It is well known a topological space is exponentiable in Top if and only if it is core compact, in the sense that any given open neighborhood $V$ of a point $x$ contains an open neighborhood $U$ of $x$ with the property that every open cover of $V$ has a finite subcover of $U$ [3]. Some characterizations of exponentiable objects in TopFuzz, and the category Tml of all topological molecular lattices were introduced in $[2,6]$. In this paper, we introduce some cartesian closed subcategories of TopFuzz. In the following, readers are suggested to refer to [I] for some categorical notions.

\section{2. preliminaries}

In this section, we first recall the definition of extra order introduced by $\mathrm{Li}$ [7]. Extra orders are useful tools to construct molecular lattices and function spaces in topological molecular lattices and topological fuzzes.

Definition 2.1. Let $P$ be a poset and $\prec$ be a binary relation on $P$.

a) $\prec$ is called an extra order, if it satisfies the following conditions:

(1) $x \prec y \Rightarrow x \leq y$,

(2) $u \leq x \prec y \leq v \Rightarrow u \prec v$. 
b) $\prec$ satisfies the interpolation property (short by INT), if $x \prec y$ implies that there exists $z \in P$ such that $x \prec z \prec y$.

Remark 2.2. If $\prec$ is an extra order on a poset $P$, then there exists a largest extra order $\prec$ over $P$ contained in $\prec$ satisfying (INT).

Definition 2.3. [7] Let $\prec$ be an extra order satisfying (INT) on a poset $P$. A subset $I$ in $P$ is called a lower-Dedekind $\prec$-cut, if it satisfies the following conditions:

(1) $\mathrm{I}$ is a lower set, that is $\downarrow I=I$.

(2) If $x \in I$, then there exists $y \in I$ such that $x \prec y$.

The set of all lower-Dedekind $\prec$-cuts in $P$ ordered by subset inclusion is denoted by $\operatorname{Low}_{\prec}(P)$. The following important result is a construction of molecular lattices using extra order.

Theorem 2.4. [7] If $\prec$ is an extra-order over $P$ satisfying (INT), then $L_{o w}(P)$ is a molecular lattice.

Remark 2.5. [6] For a complete lattice $L$, an extra order $\triangleleft$ is defined by $a \triangleleft b$ if for every subset $S \subseteq L, b \leq \vee S$ implies $a \leq s$ for some $s \in S$. If $L$ is a molecular lattice, then $\triangleleft$ satisfies the condition (INT). Also a complete lattice $L$ is a molecular lattice if and only if $b=\vee \triangleleft(b)$, where $\triangleleft(b)=\{a \in L \mid a \triangleleft b\}$.

Example 2.6. Consider the lattice $L=\{0, a, b, c, 1\}$, where $a<c, b<c$ and suppose $a$ and $b$ are incomparable. Then $L$ is molecular lattice and we have $\triangleleft(0)=\emptyset, \triangleleft(a)=\{0, a\}$, $\triangleleft(b)=\{0, b\}, \triangleleft(c)=\{0, a, b\}$ and $\triangleleft(1)=L$. By Remark [2.5, it easy to show that $\operatorname{Low}_{\triangleleft}(L)=$ $\{\emptyset,\{0, a\},\{0, b\},\{0, a, b\}, L\}$. Hence $\operatorname{Low}_{\triangleleft}(L) \cong L$.

In [II], the author used extra order to construct function spaces in topological fuzzes. For two fuzzes $\left(L_{1},{ }^{\prime}\right)$ and $\left(L_{2},{ }^{\prime}\right)$, let $\left[L_{1} \rightarrow L_{2}\right]$ denote the set of all continuous sup-preserving maps from $L_{1}$ to $L_{2}$. An extra order $\prec$ over $\left[L_{1} \rightarrow L_{2}\right]$ is defined by $f \prec g$ if $x \triangleleft y$ implies $f(x) \triangleleft g(y)$.

Definition 2.7. [II] Let $f, g \in\left[L_{1} \rightarrow L_{2}\right]$. If for every $x \in L_{1}$ the formula $\widehat{f}\left((g(x))^{\prime}\right) \leq x^{\prime}$ holds, then we say that $f$ and $g$ are parallel and denote it by $f \| g$; otherwise $f \nVdash g$.

Theorem 2.8. [11] let $\left[L_{2}^{L_{1}}\right]:=\operatorname{Low} \precsim\left[L_{1} \rightarrow L_{2}\right]$, and $:\left[L_{2}^{L_{1}}\right] \rightarrow\left[L_{2}{ }^{L_{1}}\right]$ is defined by $A^{\prime}=\bigcup\left\{B \in\left[L_{2}{ }^{L_{1}}\right]: f \nVdash g\right.$ for all $f \in A$ and $\left.g \in B\right\}$ for every $A \in\left[L_{2}{ }^{L_{1}}\right]$. Then $\left(\left[L_{2}^{L_{1}}\right],{ }^{\prime}\right)$ is a fuzz.

Theorem 2.9. [8] Top is a reflective and coreflective full subcategory of TopFuzz via the embedding power functor $\rho:$ Top $\rightarrow$ TopFuzz defined by $\rho(X, \tau)=(\rho(X), \tau)$, where $\rho(X)$ is the power set of $X$ and involution on $\rho(X)$ is the subset complement. 
Since the category Top is not cartesian closed, by Theorem [2.: it follows that TopFuzz is not a cartesian closed category. In [2], we have introduced the concept of Isbell topology on function spaces in topological fuzzes. Also for presentation of the exponentiable objects in TopFuzz we have defined an extra order $\ll$ on a topological fuzz $\left(L,{ }^{\prime}, \tau\right)$ by $a \ll b$ if for every subset $A \subseteq \tau, b \leq \vee A$ implies that there exists a finite subset $D$ of $A$ such that $a \leq \vee D$.

Definition 2.10. [2] A topological fuzz $\left(L,{ }^{\prime}, \tau\right)$ is called core compact if $b=\vee\{a \in \tau \mid a \ll b\}$ for all $b \in \tau$.

Example 2.11. Let $(X, \tau)$ be a topological space. Then $X$ is a core compact topological space if and only if $(\rho(X), \tau)$ is a core compact topological fuzz.

Remark 2.12. [II] The binary product of two topological fuzzes $\left(L_{1},{ }^{\prime}, \tau_{1}\right)$ and $\left(L_{2},{ }^{\prime}, \tau_{2}\right)$ is $\left(L_{1} \otimes L_{2},{ }^{\prime}, \tau\right)$, where $L_{1} \otimes L_{2}:=\left\{D \subseteq L_{1} \times L_{2} \mid D=\bigcup_{(x, y) \in D} \triangleleft(x) \times \triangleleft(y)\right\}, D^{\prime}=$ $\bigcap_{(x, y) \in D}\left\{\left(\triangleleft\left(x^{\prime}\right) \times \triangleleft(1)\right) \cup\left(\triangleleft(1) \times \triangleleft\left(y^{\prime}\right)\right)\right\}$ for each $D \in L_{1} \otimes L_{2}$ and $\tau$ is generated by subbase $\left\{\widehat{\pi}_{1}(x) \mid x \in \tau_{1}\right\} \cup\left\{\widehat{\pi}_{2}(y) \mid y \in \tau_{2}\right\}$, such that the projection Fuzz-maps $\pi_{1}$ and $\pi_{2}$ are defined by $\pi_{1}(D)=\bigvee\left\{x \in L_{1} \mid \exists y \in L_{2},(x, y) \in D\right\}$ and $\pi_{2}(D)=\bigvee\left\{y \in L_{2} \mid \exists x \in L_{1},(x, y) \in D\right\}$.

Theorem 2.13. [圈] Let $\left(M,{ }^{\prime}, \tau\right)$ be a topological fuzz. Then the following conditions are equivalent:

(1) $\left(M,{ }^{\prime}, \tau\right)$ is exponentiable in TopFuzz, and for every topological fuzz $L$, the exponential topology on $\left[L^{M}\right]$ is the Isbell topology.

(2) $\left(M,{ }^{\prime}, \tau\right)$ is core compact.

(3) The mapping ev $:\left(\left[L^{M}\right],{ }^{\prime}, \eta\right) \otimes\left(M,{ }^{\prime}, \tau\right) \rightarrow\left(L,{ }^{\prime}, \mu\right)$ defined by ev $(D)=\vee\{f(x) \mid f \in$ $A,(A, x) \in D\}$ is a continuous fuzz-map for every topological fuzz $\left(L,{ }^{\prime}, \mu\right)$.

If $M$ is an exponentiable topological fuzz, then $\left[L^{M}\right]$ equipped with the exponential topology is denoted by $L^{M}$.

\section{3. $\mathcal{C}$-generated topological fuzzes}

Let $L_{1}$ and $L_{2}$ be topological fuzzes and let $C\left(L_{1}, L_{2}\right)$ denote the set of all continuous fuzzmaps from $L_{1}$ to $L_{2}$. The transpose $\bar{f}: M \rightarrow\left[L_{2}^{L_{1}}\right]$ of a fuzz-map $f: M \otimes L_{1} \rightarrow L_{2}$ is defined by $\bar{f}(a)=f_{a}$, where $f_{a} \in\left[L_{2}^{L_{1}}\right]$ is given by $f_{a}(x)=f(\triangleleft(a) \times \triangleleft(x))$.

By definition of the exponentiable objects in terms of adjoint situations [T], we have a topological fuzz $L_{1}$ is exponentiable if and only if for every topological fuzz $L_{2}$ there exists a topology on $\left[L_{2}^{L_{1}}\right]$ such that for any topological fuzz $M$, the association $f \leftrightarrow \bar{f}$ is a bijection from $C\left(M \otimes L_{1}, L_{2}\right)$ to $C\left(M,\left[L_{2}{ }^{L_{1}}\right]\right)$.

Lemma 3.1. The product of two exponentiable topological fuzzes is exponentiable. 
Proof. Let $L_{1}, L_{2}$ be two exponentiable topological fuzzes. Then for arbitrary topological fuzzes $M$ and $N$, the following bijections hold: $C\left(M \otimes\left(L_{1} \otimes L_{2}\right), N\right) \cong C\left(\left(M \otimes L_{1}\right) \otimes L_{2}, N\right)$ $\cong C\left(M \otimes L_{1}, N^{L_{2}}\right) \cong C\left(M,\left(N^{L_{2}}\right)^{L_{1}}\right)$. Moreover, $\left[\left(N^{L_{2}}\right)^{L_{1}}\right] \cong\left[N^{L_{1} \otimes L_{2}}\right]$. Hence the exponential topology on $\left[\left(N^{L_{2}}\right)^{L_{1}}\right]$ induces an exponential topology on $\left[N^{L_{1} \otimes L_{2}}\right]$.

Corollary 3.2. The product of two core compact topological fuzzes is core compact.

Lemma 3.3. The coproduct of a family of exponentiable topological fuzzes is exponentiable.

Proof. Let $\left\{L_{i}\right\}_{i \in I}$ be a family of exponentiable topological fuzzes and $M$ be an arbitrary topological fuzz. By the natural bijection $\bigotimes_{i}\left[M^{L_{i}}\right] \cong\left[M^{L_{i}}\right]$, it follows that $\bigotimes_{i}\left[M^{L_{i}}\right]$ induces an exponential topology on $\left[M \amalg_{i} L_{i}\right]$, where $\coprod_{i} L_{i}$ is the coproduct of $\left\{L_{i}\right\}_{i \in I}$.

Definition 3.4. Let $\mathcal{C}$ be a fixed collection of topological fuzzes, which are called generating topological fuzzes. A probe over topological fuzz $L$ is a continuous fuzz-map from one of the generating topological fuzzes to $L$. The $\mathcal{C}$-generated topology $\mathcal{C} L$ on a topological fuzz $L$ is the final topology of the probes over $L$, that is, the finest topology making all probes continuous. We say that a topological fuzz $L$ is $\mathcal{C}$-generated, if $L=\mathcal{C} L$. The category of $\mathcal{C}$-generated topological fuzzes and continuous fuzz-maps between them is denoted by $\mathbf{T o p F u z z} \mathbf{C}_{\mathcal{C}}$.

Lemma 3.5. The following statements hold.

(1) Every generating topological fuzz is $\mathcal{C}$-generated.

(2) $\mathcal{C}$-generated topological fuzzes are closed under the formation of quotients.

(3) $\mathcal{C}$-generated topological fuzzes are closed under the formation of coproducts.

(4) Every $\mathcal{C}$-generated topological fuzz is a quotient of a coproduct of generating topological fuzzes.

Proof. (1) : Consider the identity probe, then the result follows.

(2) : Let $f: L_{1} \rightarrow L_{2}$ be a quotient fuzz-map and $L_{1}$ be $\mathcal{C}$-generated. Then the composite probes $f \circ p: C \rightarrow L_{2}$ suffice to generate topology of $L_{2}$, where $p: C \rightarrow L_{1}$ varies over all probes of $L_{1}$.

(3) : The proof is similar to $(2)$.

(4) : Let $L$ be a $\mathcal{C}$-generated topological fuzz and $I$ be the set of non-open elements of $L$. Then by definition of final topology, for each $i \in I$ there exists a probe $p_{i}: C_{i} \rightarrow L$ which $\widehat{p}_{i}(i)$ is non-open. By the choice of probes, an element $a$ of $L$ is open if and only if $\widehat{p}_{i}(a)$ is open for all $i \in I$, that is, the topology of $L$ is just the final topology of the family $\left\{p_{i}: C_{i} \rightarrow L\right\}_{i \in I}$. We can enlarge this family, if necessary, by including all constant maps from some nonempty generating fuzzes, to get sure that all elements of $L$ are covered by 
probes. Let $\left(J_{i}: C_{i} \rightarrow S\right)_{i \in I}$ be the coproduct of the topological fuzzes $\left\{C_{i}\right\}_{i \in I}$. According to the universal property of coproducts, there is a unique fuzz-map $q: S \rightarrow L$ such that $q \circ J_{i}=p_{i}$ for all $i \in I$. Again by the universal property, a function $f: L \rightarrow M$ is continuous if and only if $f \circ q: S \rightarrow M$ is continuous, which shows that $q$ is a quotient fuzz-map.

Definition 3.6. [4] An element $a$ of a topological fuzz $L$ is called compact if $a \ll a$, and $L$ is called compact if every its closed element is compact.

Definition 3.7. [5] A topological fuzz $\left(L,{ }^{\prime}, \tau\right)$ is called Hausdorff $\left(T_{2}\right)$ if the following conditions hold:

(1) Every $a \in L$ can be written in the form $a=\bigwedge_{i \in I} \bigvee_{j \in J_{i}} u_{i j}$, where $u_{i j}$ is open or closed for every $i \in I$ and $j \in J_{i}$.

(2) Every $a \in L$ can be written in the form $a=\bigwedge_{i \in I} \bigvee_{j \in J_{i}} u_{i j}=\bigwedge_{i \in I} \bigvee_{j \in J_{i}} \bar{u}_{i j}$, where $u_{i j}$ is open for every $i \in I$ and $j \in J_{i}$, and $\bar{u}_{i j}$ denote the closure of $u_{i j}$.

Definition 3.8. A topological fuzz $\left(L,{ }^{\prime}, \tau\right)$ is said to be locally compact, if for each $b \in \tau$ and each $m \in \triangleleft(b)$, then there exist $a \in \tau$ and $c \in L$ compact such that $m \leq a \leq c \leq b$.

Clearly, if $(X, \tau)$ is a locally compact (compact) topological space, then $(\rho(X), \tau)$ is a locally compact (compact) topological fuzz. If $a \leq c \leq b$ such that $c$ is compact, then we have $a \ll b$. This implies that every locally compact topological fuzz is core compact.

Lemma 3.9. Every compact Hausdorff topological fuzz is locally compact.

Proof. Let $\left(L,{ }^{\prime}, \tau\right)$ be a compact Hausdorff topological fuzz, $b \in \tau$ and $m \in \triangleleft(b)$. Since $\triangleleft$ satisfies the condition (INT), it follows that there exists $d \in L$ such that $m \triangleleft d \triangleleft b$. By assumption, $d=\bigwedge_{i \in I} \bigvee_{j \in J_{i}} u_{i j}=\bigwedge_{i \in I} \bigvee_{j \in J_{i}} \bar{u}_{i j}$, where $u_{i j}$ is open. Then for each $i \in I$, $d \leq \bigvee_{j \in J_{i}} u_{i j} \leq \bigvee_{j \in J_{i}} \bar{u}_{i j}$. Thus $m \leq v_{i} \leq \bar{v}_{i} \leq \bigvee_{j \in J_{i}} \bar{u}_{i j}$, where $v_{i}=u_{i j}$ for some $j \in J_{i}$. Then $m \leq \bigwedge_{i \in I} v_{i} \leq \bigwedge_{i \in I} \bar{v}_{i} \leq d \leq b$. Hence $b^{\prime} \leq \bigvee_{i \in I} \bar{v}_{i}^{\prime}$. By compactness, there exists a finite subset $F$ of $I$ such that $\bigwedge_{i \in F} \bar{v}_{i} \leq b$. Let $a=\bigwedge_{i \in F} v_{i}$. Then $a \in \tau$ and $m \leq a \leq \bar{a} \leq b$, as desired.

Example 3.10. Consider the lattice $L=[0,1]$ with usual order. Define the involution ' on $L$ by $a^{\prime}=1-a$ for every $a \in L$. If $\tau=L$, then $\left(L,^{\prime}, \tau\right)$ is a topological fuzz. Since $a \triangleleft b$ implies $a \ll b$, By Remark [2.5, it follows that $b=\vee \triangleleft(b)=\vee\{a \in \tau \mid a \ll b\}$ for every $b \in \tau$. Thus $L$ is a core compact Hausdorff topological fuzz. On the other hand, every nonzero element of $L$ is not compact, so $L$ is neither locally compact nor compact.

Definition 3.11. A topological fuzz is called: 
Alg. Struc. Appl. Vol. 6 No. 1 (2019) 23-33.

(1) core compactly generated, if $\mathcal{C}$ consists of core compact topological fuzzes,

(2) compactly generated, if $\mathcal{C}$ consists of compact Hausdorff topological fuzzes.

Corollary 3.12. The following statements hold.

(1) A topological fuzz is core compactly generated if and only if it is a quotient of a core compact topological fuzz.

(2) A topological fuzz is compactly generated if and only if it is a quotient of a compact Hausdorff topological fuzz.

Proof. Since the class of core compact topological fuzzes and compact Hausdorff topological fuzzes are closed under the coproducts and by Lemma $\mathbf{3 . 5}$, it follows directly.

Definition 3.13. A class $\mathcal{C}$ of generating topological fuzzes is called productive if each element of $\mathcal{C}$ is exponentiable, and the topological fuzz product of any two generating spaces is $\mathcal{C}$ generated.

Example 3.14. The class of all core compact topological fuzzes and the class of all compact Hausdorff topological fuzzes are productive.

Definition 3.15. A sup-preserving map $f: L_{1} \rightarrow L_{2}$ is called $\mathcal{C}$-continuous if $f \circ p: C \rightarrow L_{2}$ is continuous for each probe $p: C \rightarrow L_{1}$.

It is clear that every continuous fuzz-map is $\mathcal{C}$-continuous and for fuzz-maps defined on $\mathcal{C}$-generated topological fuzzes, the $\mathcal{C}$-continuity coincides with continuity. The topological fuzzes and $\mathcal{C}$-continuous fuzz-maps between them form a category which is denoted by Map . It is easy to show that the identity fuzz-map $C L \rightarrow L$ is an isomorphism in $\mathbf{M a p}_{\mathcal{C}}$.

Lemma 3.16. The functor $\mathcal{C}: \mathbf{M a p}_{\mathcal{C}} \rightarrow$ TopFuzz $_{\mathcal{C}}$ that sends a topological fuzz $L$ to $\mathcal{C} L$ and a $\mathcal{C}$-continuous map to itself is an equivalence of categories.

Proof. TopFuzz $\mathbf{T}_{\mathcal{C}}$ is a full subcategory of $\operatorname{Map}_{\mathcal{C}}$, because a topological fuzz $L$ is $\mathcal{C}$-generated if and only if continuity of a function defined on $L$ is equivalent to $\mathcal{C}$-continuity. On the other hand, every topological fuzz is isomorphic in $\mathbf{T o p F u z z} \mathbf{C}_{\mathcal{C}}$ to an object of $\mathbf{M a p}_{\mathcal{C}}$. Thus the result holds.

Lemma 3.17. Map $_{\mathcal{C}}$ has finite products and they are the same as in TopFuzz.

Proof. It is enough to show that $L_{1} \otimes L_{2}$ has the universal property, where $L_{1}$ and $L_{2}$ are topological fuzzes. Since the projection fuzz-maps $\pi_{1}$ and $\pi_{2}$ are continuous, they are $\mathcal{C}$ continuous. Let $f_{i}: M \rightarrow L_{i}$ be an arbitrary $\mathcal{C}$-continuous fuzz-map for $i=1,2$. Then there 
exists a unique fuzz-map $f: M \rightarrow L_{1} \otimes L_{2}$ such that $f_{i}=\pi \circ f$. Now, we show that $f$ is $\mathcal{C}$-continuous. Let $p: C \rightarrow M$ be a probe. It is enough to prove that $\pi_{i} \circ f \circ p$ is continuous. But $\pi_{i} \circ f \circ p=f_{i} \circ p$, which is continuous by $\mathcal{C}$-continuity of $f_{i} \cdot$

Remark 3.18. Let $\mathcal{C}$ be an arbitrary collection of topological fuzzes. For $\mathcal{C}$-generated topological fuzzes $L_{1}$ and $L_{2}$, we write $L_{1} \otimes_{\mathcal{C}} L_{2}$ for binary product in the category TopFuzz $\mathbf{C}_{\mathcal{C}}$ Trivially, if the topological fuzz product $L_{1} \otimes L_{2}$ is itself $\mathcal{C}$-generated, then $L_{1} \otimes_{\mathcal{C}} L_{2}=L_{1} \otimes L_{2}$. Moreover, since the functor $\mathcal{C}: \mathbf{M a p}_{\mathcal{C}} \rightarrow \mathbf{T o p F u z z}_{\mathcal{C}}$ is an equivalence of categories, it follows that $L_{1} \otimes_{\mathcal{C}} L_{2}=\mathcal{C}\left(L_{1} \otimes L_{2}\right)$.

Recall that an element $a$ of a lattice $L$ is called coprime, if $a \leq b \vee c$ implies $a \leq b$ or $a \leq c$, for every $b, c \in L$. We denote by $C P(L)$ the set of all nonzero coprime elements of $L$. Nonzero coprime elements are also called molecules. It is well known that if $L$ is a molecular lattice, then $C P(L)$ is a join generating base for $L$, i.e., every element of $L$ is a join of some elements of $C P(L)[9]$.

Lemma 3.19. Let $f: M \otimes L_{1} \rightarrow L_{2}$ be a $\mathcal{C}$-continuous fuzz-map. Then for each $p \in C P(M)$ the fuzz-map $f_{p}: L_{1} \rightarrow L_{2}$ defined by $f_{p}(a)=f(\triangleleft(p) \times \triangleleft(a))$, is $\mathcal{C}$-continuous.

Proof. For each $p \in C P(M)$ the fuzz-map $g_{p}: L_{1} \rightarrow M \otimes L_{1}$ defined by $g_{p}(a)=\triangleleft(p) \times \triangleleft(a)$ is $\mathcal{C}$-continuous, because it is continuous. Since $f \circ g_{p}=f_{p}$, the result follows directly.

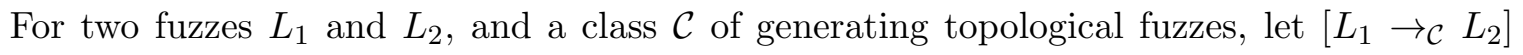
denote the set of all $\mathcal{C}$-continuous sup-preserving maps from $L_{1}$ to $L_{2}$. Define an extra order $\prec_{\mathcal{C}}$ over $\left[L_{1} \rightarrow_{\mathcal{C}} L_{2}\right]$ by $f \prec_{\mathcal{C}} g$ if whenever $x \triangleleft y$, then $f(x) \triangleleft g(y)$.

Now, let $\left[L_{2}^{L_{1}}\right]_{\mathcal{C}}=\operatorname{Low}_{\prec}\left[L_{1} \rightarrow_{\mathcal{C}} L_{2}\right]$, and ' $:\left[L_{2}{ }^{L_{1}}\right]_{\mathcal{C}} \rightarrow\left[L_{2}{ }^{L_{1}}\right]_{\mathcal{C}}$ is defined by $A^{\prime}=\bigcup\{B \in$ $\left[L_{2}{ }^{L_{1}}\right]_{\mathcal{C}}: f \nVdash g$ for all $f \in A$ and $\left.g \in B\right\}$ for every $A \in\left[L_{2}{ }^{L_{1}}\right]_{\mathcal{C}}$. Then it is easy to show that $\left(\left[L_{2}^{L_{1}}\right]_{\mathcal{C}},{ }^{\prime}\right)$ is a fuzz.

Let $f: M \otimes L_{1} \rightarrow L_{2}$ be a $\mathcal{C}$-continuous fuzz-map. Then we have a function $\bar{f}: M \rightarrow\left[L_{2}{ }^{L_{1}}\right]_{\mathcal{C}}$ such that $\bar{f}(x)=f_{x}$. Suppose that any element of $\mathcal{C}$ is exponentiable. Now, we define a topology on $\left[L_{2}{ }^{L_{1}}\right]_{\mathcal{C}}$. Each probe $p: C \rightarrow L_{1}$ induces a function $T_{p}:\left[L_{2}{ }^{L_{1}}\right]_{\mathcal{C}} \rightarrow L_{2}^{C}$ defined by $T_{p}(g)=g \circ p$. We endow $\left[L_{2}{ }^{L_{1}}\right]_{\mathcal{C}}$ with initial topology of the family of functions that arise in this way, obtaining a topological fuzz $\left(L_{2}{ }^{L_{1}}\right)_{\mathcal{C}}$. Thus for any topological fuzz $L$, a function $h: L \rightarrow\left(L_{2}{ }^{L_{1}}\right)_{\mathcal{C}}$ is continuous if and only if $T_{p} \circ h: L \rightarrow L_{2}^{C}$ is continuous for each probe $p: C \rightarrow L_{1}$.

Lemma 3.20. Let $\mathcal{C}$ be a class of productive topological fuzzes. 
(1) A fuzz-map $h: L \rightarrow\left(L_{2}{ }^{L_{1}}\right)_{\mathcal{C}}$ is continuous if and only if the fuzz-map $g: L \otimes C \rightarrow L_{2}$ defined by $g(\triangleleft(l) \times \triangleleft(c))=(h(l))(p(c))$ is continuous, for each probe $p: C \rightarrow L_{1}$.

(2) The transpose $\bar{f}: M \rightarrow\left(L_{2}{ }^{L_{1}}\right)_{\mathcal{C}}$ of a function $f: M \otimes L_{1} \rightarrow L_{2}$ is $\mathcal{C}$-continuous if and only if the function $f \circ(p \otimes q): C_{1} \otimes C_{2} \rightarrow L_{2}$ is continuous, for all probes $p: C_{1} \rightarrow M$ and $q: C_{2} \rightarrow L_{1}$.

(3) A function $f: M \otimes L_{1} \rightarrow L_{2}$ is $\mathcal{C}$-continuous if and only if the function $f \circ(p \otimes q)$ : $C_{1} \otimes C_{2} \rightarrow L_{2}$ is continuous, for all probes $p: C_{1} \rightarrow M$ and $q: C_{2} \rightarrow L_{1}$.

Proof. (1) : For each probe $p: C \rightarrow L_{1}$, a function $h: L \rightarrow\left(L_{2}{ }^{L_{1}}\right)_{\mathcal{C}}$ is continuous if and only if $T_{p} \circ h: L \rightarrow L_{2}^{C}$ is continuous. Since $T_{p} \circ h$ is the transpose of $g: L \otimes C \rightarrow L_{2}$ and $C$ is exponentiable in TopFuzz, we have $T_{p} \circ h$ is continuous if and only if $g$ is continuous.

(2) : The function $\bar{f}$ is $\mathcal{C}$-continuous if and only if $\bar{f} \circ p: C_{1} \rightarrow\left(L_{2}{ }^{L_{1}}\right)_{\mathcal{C}}$ is continuous for each probe $p: C_{1} \rightarrow M$. According to the previous part $\bar{f} \circ p$ is continuous if and only if $g: C_{1} \otimes C_{2} \rightarrow L_{2}$ is continuous for each probe $q: C_{2} \rightarrow L_{1}$, where $g(\triangleleft(x) \times \triangleleft(y))=((\bar{f} \circ p)(x))(q(y))$. Since $((\bar{f} \circ p)(x))(q(y))=f(\triangleleft(p(x)) \times \triangleleft(q(y)))=f \circ(p \otimes q)(\triangleleft(x) \times \triangleleft(y))$, the result follows. (3) : Since $\mathcal{C}$ is productive, $C_{1} \otimes C_{2}$ is $\mathcal{C}$-generated. Therefore, $f \circ(p \otimes q)$ is continuous if and only if it is $\mathcal{C}$-continuous, that is, $f \circ(p \otimes q) \circ s$ is continuous for each probe $s: C_{3} \rightarrow C_{1} \otimes C_{2}$. But $f \circ(p \otimes q)$ is continuous, so is $f \circ(p \otimes q) \circ s$.

For converse, let $r: C \rightarrow M \otimes L_{1}$ be an arbitrary probe over $M \otimes L_{1}$. We will show that $f \circ r$ is continuous. The probes $p: C \rightarrow M$ and $q: C \rightarrow L_{1}$ obtained by composing $r$ with the projections. Since $d: C \rightarrow C \otimes C$ is continuous, then $f \circ(p \otimes q) \circ d=f \circ r: C \rightarrow L_{2}$ is continuous. Thus $f$ is $\mathcal{C}$-continuous.

Now, by Lemmas [3.16 and [3.20, we have the following main result.

Theorem 3.21. If $\mathcal{C}$ is a class of productive topological fuzzes, then $\mathbf{M a p}_{\mathcal{C}}$ is cartesian closed and so is TopFuzz $_{\mathcal{C}}$.

Corollary 3.22. Core compactly generated and compactly generated topological fuzzes are cartesian closed subcategories of TopFuzz.

The following Example shows that the converse of the previous Theorem need not be true in general.

Example 3.23. Let $\mathcal{C}$ be the collection of all exponentiable topological fuzzes and $\mathcal{D}=$ TopFuzz $_{\mathcal{C}}$. Then it is easy to show that a topological fuzz is $\mathcal{D}$-generated if and only if it is quotient of a $\mathcal{C}$-generating topological fuzz. Thus $\operatorname{TopFuzz}_{\mathcal{D}}=\mathbf{T o p F u z z}_{\mathcal{C}}$, and hence it is cartesian closed. But we prove that $\mathcal{D}$ is not productive. In [3] (Example 5.1), it is shown that 
there exist exponentiable spaces $X$ and $Y$ in Top such that the topological product $X \times Y$ does not generated by the collection of all exponentiable spaces in Top. By Theorem [2.9, we have $\rho(X \times Y) \cong \rho(X) \otimes \rho(Y)$. Hence by Example [2.], the topological fuzz product $\rho(X) \otimes \rho(Y)$ does not generated by $\mathcal{D}$.

Remark 3.24. Let 1 be the one point topological fuzz. Since discreet fuzzes are 1-generated, it follows that they form a cartesian closed category. This of course amounts to the familiar fact that the category Fuzz is cartesian closed [I]].

\section{Conclusion}

It is well known that the category TopFuzz of all topological fuzzes is both complete and cocomplete and some categorical structures of it were introduced by many authors. Since the category Top of all topological spaces is a reflective and coreflective subcategory of TopFuzz, it follows that TopFuzz is not a cartesian closed category. In our previous work, we have introduced some characterizations of the exponentiable topological fuzzes. In this paper, we have presented some cartesian closed subcategories of TopFuzz. For a class $\mathcal{C}$ of topological fuzzes, we have defined the concepts as generated topological fuzz, productive class and the category of $\mathcal{C}$-generated topological fuzzes. As an important result we have proved that if $\mathcal{C}$ is productive, then the category of $\mathcal{C}$-generated topological fuzzes is cartesian closed. In particular, we have shown that the category of core compactly generated topological fuzzes and the category of compactly generated topological fuzzes are cartesian closed subcategories of TopFuzz.

\section{ACKNOWLEDGMents}

We are very grateful to the referee(s) for the careful reading of the paper and for the useful comments.

\section{REFERENCES}

[1] J. Adamek, H. Herrlich and G. E. Strecker, Abstract and concrete categories, John Wiely and Sons Inc., New York, 1990.

[2] M. Akbarpour and GH. Mirhosseinkhani, Exponentiable objects in some categories of topological molecular lattices, Hadronic Journal, 40 (2017), 327-344.

[3] M. Escardo, J. Lawson and A. Simpson, Comparing cartesian closed categories of (core)compactly generated spaces, Topology Appl., 143 (2004), 105-145.

[4] B. Hutton, Products of fuzzy topological spaces, Topology Appl., 11 (1980), 59-67.

[5] B. Hutton and I. Reilly, Separation axioms in fuzzy topological spaces, Fuzzy Sets and Systems, 3 (1980), 93-104. 
[6] Y.M. Li, Exponentiable objects in the category of topological molecular lattices, Fuzzy Sets and Systems, 104 (1999), 407-414.

[7] Y.M. Li, Generalized (S,I)-complete free completely distributive lattices generated by posets, Semigroup Forum, 57 (1998), 240-248.

[8] Y.M. Li and Z.H. Li, Top is a reflective and coreflective subcategory of fuzzy topological spaces, Fuzzy Sets and Systems, 116 (2000), 429-432.

[9] G.J. Wang, Theory of topological molecular lattices, Fuzzy Sets and Systems, 47 (1992), $351-376$.

[10] G.J. Wang, Order-homomorphisms on fuzzes, Fuzzy Sets and Systems, 12 (1984), 281288.

[11] Z. Yang, The cartesian closedness of the category Fuzz and function spaces on topological fuzzes, Fuzzy Sets and Systems, 61 (1994), 341-351.

\section{Mahboobeh Akbarpour}

Department of mathematics,

University of Hormozgan

Bandarabbas, Iran.

b. akbarpour66@gmail.com

\section{Ghasem Mirhosseinkhani}

Department of mathematics and Computer Sciences

Sirjan University of Technology

Sirjan, Iran.

gh.mirhosseini@yahoo.com, gh.mirhosseini@sirjantech.ac.ir 